\title{
Time Scales of Inoculum Production and the Dynamics of the Epidemic
}

\author{
Francis J. Ferrandino
}

Department of Plant Pathology and Ecology, The Connecticut Agricultural Experiment Station, P.O. Box 1106, New Haven 06504. Accepted for publication 26 April 2012.

For the past half century, the latent period and the infectious period introduced by Vanderplank $(28,29)$ have dominated any discussion of plant disease epidemics. These two time scales were defined in a relatively simple model of inoculum production, in which every infection goes through a latent period of time, $p$, before becoming infectious, and every lesion synchronously produces progeny at a constant rate, $R$ (Vanderplank's "Basic Infection Rate") (28-30), for a specific infectious period of time, $i$. Thus, in Vanderplank's model, the reproduction curve is given by a square temporal flush of progeny production between time $p$ and time $p+i$. Alternatively, the first two temporal moments of the reproduction curve provide a pair of reproductive time scales: the mean delay time between infection and progeny production, $\mu$, and the standard deviation about this mean, $\sigma$. The use of temporal moments to describe population growth dates back to the inception of population dynamics (4) and has become an integral part of the literature on this subject (24). In bacteriology, the measured doubling time of a population has been expressed in terms of the mean time and variance between individual mitotic events, $\mu$ and $\sigma$, for decades (22). Metz and Diekmann (21) presented a number of approximations for the initial infection rate in terms of the temporal moments of the sporulation curve. Which set of time scales does one choose to describe the reproduction, and does it matter?

For the simple case described above, in which every infection follows exactly the same reproductive time course, the values of $\mu$ and $\sigma$ are uniquely determined by the values of $p$ and $i$. In reality, there is considerable variability in these time scales among individual infections because of several factors, including somatic differences in host tissue and differences in microclimate within the plant canopy, as well as the inherent range of virulence within the pathogen population. For such a dynamic situation, (i) how does one define "average" values for the latent period and the infectious period, (ii) how can these values be experimentally determined, and (iii) how do these values relate to $\mu$ and $\sigma$ ?

Population averages of the latent period and the infectious period. By nature, the latent period, $p$, and the infectious period, $i$, are defined over the population of parental infections ("mother lesions"). Thus, when variation is present, the logical way to define a population average value for $p$ (denoted in this letter by $\bar{p}$ ) or $i$ (denoted in this letter by $\vec{l}$ ) is to take the sum of the values for each individual in the population and divide by the number of individuals. Note that the contribution of each infection is weighted equally in this process.

Corresponding author: F. J. Ferrandino

E-mail address: francis.ferrandino@po.state.ct.us

* The $\boldsymbol{e}$-Xtra logo stands for "electronic extra" and indicates that the online version contains supplemental material not available in the print version.

http://dx.doi.org/10.1094/PHYTO-03-12-0051-LE

(C) 2012 The American Phytopathological Society
The literature contains experimentally measured sporulation curves for many plant pathogens $(1,2,6,7,17,19,23,25,27)$. The latent period is usually defined as the initial period of time during which no inoculum is produced (here denoted by $p_{0}$ ). This is the latent period of the most precocious infection and may not be the best descriptor for the behavior of the bulk of the infected population (30). For example, the daily production of sporangia of Pseudoperonospora cubensis (cucumber downy mildew) (3) per infected cucumber cotyledon is plotted versus time after inoculation for four incubation night-and-day temperature ranges (Fig. 1). The sporulation curve for the 20 to $30^{\circ} \mathrm{C}$ temperature range (Fig. 1, arrow) indicates an observed value for $p_{0}$ of between 3 and 4 days, because 3,000 sporangia were collected on the fourth day. However, this does not mean that all of the initial infections were sporulating by day 4 . In fact, the daily spore production rate increases 20 -fold to 60,000 sporangia/day by day 6 . This suggests that the population average for the latent period (denoted in this letter by $\bar{p}$ ) is at least 5 days; however, there is no clear method to extract this information from the experimental data. This delay between $p_{0}$ and $\bar{p}$ becomes more evident as incubation temperature decreases (Fig. 1).

The extraction of the infectious period and its distribution from an experimental curve is more nebulous; in fact, it is totally arbitrary. All we can glean from the sporulation curve is that the maximum value for the infectious period, $i_{\max }$, must be less than the last day of sporulation minus the minimum latent period, $p_{0}$; which, for the sporulation curve for the 20 to $30^{\circ} \mathrm{C}$ temperature range (Fig. 1, arrow) results in $i_{\max }<8$ days -3 days $=5$ days. On the other hand, the mean time of spore production, $\mu$, and the standard deviation, $\sigma$, can be estimated directly from the experimental sporulation curve.

In comparing a number of theoretical models for spore production, Segarra et al. (26) suggested that the proper way to compare these various models was to assure that "The average duration of the infection cycle is $p+i \ldots$. " was the same for all distributions under consideration (26) (Fig. 1, caption). Recently, Cunniffe et al. (3) also used latent and infectious periods in favor of the temporal moments to match parameters between model frameworks. However, the simple use of a population average for latent period and infectious period, $\bar{p}$ and $\bar{l}$, is problematical. As I will show in this letter, the shape of the resulting reproduction curve and, hence, the time course of the ensuing epidemic do not depend on average values of $p$ and $i$ alone but are also critically dependent on the variance of these values about their respective means and the covariance between them. Thus, whether the reproduction curve is assumed a priori or experimentally determined, this function does not provide enough information to simultaneously determine the distributions of both the latent period and the infectious period over the pathogen population. This leads to the anomaly that different models of spore production, which have the same mean values for $p$ and $i$, can result in markedly 
different epidemics. On the other hand, $\mu$ and $\sigma$ are defined in terms of the next generation of infections ("daughter lesions"). Thus, the calculation of $\mu$ and $\sigma$ is implicitly weighted by the total number of progeny produced by each individual. Mother lesions with a larger value for the infectious period produce more offspring and are weighted more heavily in the calculation of the moments. My goal in this letter is to elucidate the problems associated with population-averaged reproductive time scales and to show that the temporal moments of the reproduction curve, whether they are determined theoretically or experimentally, provide a less ambivalent description of the dynamics of the resulting epidemic.

Vanderplank's model. Let us consider Vanderplank's $(28,29)$ relatively simple epidemic model based on a square pulse (SP) of inoculum, such that

$$
\Re_{S P}(\tau, p, i)=\left\{\begin{array}{cc}
0 & \tau<p \\
R & p \leq \tau \leq p+i \\
0 & p+i<\tau
\end{array}\right.
$$

where $\Re_{S P}(\tau, p, i)$ is the reproduction curve (the potential number of daughter lesions produced per mother lesion per unit time), $\tau$ is the age of the mother lesion (measured from initial infection), $p$ is the latent period (the period of time before infected tissue becomes infectious), $i$ is the infectious period, and $R$ is the basic infection rate (daughter lesions per unit time) (30). The reproduction curve, $\Re_{S P}(\tau, p, i)$, includes the effects of latency, the rate of daughter lesion production over the infectious period, and the removal of infectious tissue from the system by senescence or defoliation of the host tissue.

Temporal moments of the reproduction curve. In general, the mean time between generations of the pathogen, $\mu$, and the variance, $\sigma^{2}$, about this mean are defined by

$$
\begin{gathered}
\mu=\frac{\int_{0}^{\infty} \tau \cdot \Re(\tau) d \tau}{\int_{0}^{\infty} \Re(\tau) d \tau} \\
\sigma^{2}=\frac{\int_{0}^{\infty}(\tau-\mu)^{2} \cdot \Re(\tau) d \tau}{\int_{0}^{\infty} \Re(\tau) d \tau}
\end{gathered}
$$

which, for equation 1 , yields $\mu_{\mathrm{SP}}=p+i / 2$ and $\sigma_{\mathrm{SP}}^{2}=i^{2} / 12$, the standard moments of a uniform distribution ("top hat" distribution). Note that the subscript "SP" denotes that the reproduction curve used is Vanderplank's SP model given by equation 1 .

Now let us consider the case where there is a population of $n$ lesions sporulating according to equation 1 , such that the $k$ th cohort is characterized by a latent period, $p_{\mathrm{k}}$, and an infectious period $i_{\mathrm{k}}$. In general, each lesion may have its own basic infection rate, $R_{\mathrm{k}}$. For simplicity, we assume that the value of $R$ is the same for all values of $k$. This is in keeping with the theoretical spore production models proposed by Segarra et al. (26). The sporulation curve of this ensemble, $\mathfrak{R}_{\text {ens }}(\tau)$, can be expressed as an average over the $n$ cohorts:

$$
\Re_{\text {ens }}(\tau)=\frac{1}{n} \sum_{k=1}^{n} \Re_{S P}\left(\tau, p_{k}, i_{k}\right)
$$

By inserting equation 3 into equation 2 and interchanging the order of the summation and the integration, we obtain the mean time (as an ensemble average) between generations of the pathogen, $\mu_{e n s}$, and the variance, $\sigma_{e n s}^{2}$, about this mean for the ensemble in equation 3:

$$
\mu_{\text {ens }}=\frac{\frac{1}{n} \sum_{k=1}^{n}\left(i_{k} p_{k}+\frac{i_{k}^{2}}{2}\right)}{\frac{1}{n} \sum_{k=1}^{n} i_{k}}=\bar{p}+\frac{\bar{i}}{2}+\frac{\operatorname{cov}(p, i)}{\bar{i}}+\frac{\sigma_{i}^{2}}{2 \bar{i}}
$$

$\sigma_{\text {ens }}^{2}=\frac{\bar{i}^{2}}{12}+\sigma_{p}^{2}+\frac{\sigma_{i}^{2}}{2}\left(1-\frac{\sigma_{i}^{2}}{2 \bar{i}^{2}}\right)+\frac{S k_{i} \sigma_{i}^{3}}{3 \bar{i}}+\operatorname{cov}(p, i)\left(1-\frac{\sigma_{i}^{2}}{\bar{i}^{2}}-\frac{\operatorname{cov}(p, i)}{\bar{i}^{2}}\right)+\frac{\overline{p^{\prime 2} i^{\prime}}}{\bar{i}}+\frac{\overline{p^{\prime} i^{\prime 2}}}{\bar{i}}$

where $\bar{p}$ is the mean latent period, $\bar{l}$ is the mean infectious period, $p^{\prime}$ and $i^{\prime}$ are the deviations from these means, $\sigma_{p}{ }^{2}$ and $\sigma_{i}{ }^{2}$ are the variances about these means, $\operatorname{cov}(p, i)$ is the covariance between the latent and infectious periods, $S k_{i}$ is the skewness of the infectious period distribution, and the other third-order moments (the last two terms) are explicitly defined in the supplemental material available online, which also includes details of the analysis.

The above analysis (equation 4) clearly illustrates the point that specification of average values for $p$ and $i$, alone, does not provide sufficient information to determine the reproduction curve. In fact, the complexity of the expressions for the first two temporal moments of the reproduction curve (equation 4) in terms of latent and infectious periods challenges the usefulness of these time scales when variability is present.

A thought experiment. The algebraic complexity of equation 4 may distract a reader from the simple conclusion of the above derivation. What is most important is that, when there is a collection of infections that have variable latent and infectious periods, these concepts are no longer meaningful in describing the resultant epidemic. To illustrate this fact, I propose the following thought experiment.

Consider Vanderplank's SP model with latent period $p=6$ days and infectious period $i=10$ days (Fig. 2A). Thus, $\mu_{\mathrm{SP}}=p+i / 2=$ 11 days and $\sigma_{\mathrm{SP}}{ }^{2}=i^{2} / 12=100 / 12=8.33$ days $^{2}$ (Table 1 ). One can also think of this reproduction curve as consisting of 10 different cohorts with identical parameters (Fig. 3A). If one holds $i$ constant at 10 days and varies $p$ about that original value of 6 days (Fig. 3B), $\bar{p}$ can be kept constant. The ensemble mean, $\mu_{\text {ens }}$, remains the same ( 11 days) but the variance, $\sigma^{2}{ }_{\text {ens }}$, is doubled to a value of 16.67 days $^{2}$. The "leaning tower"-type reproduction curve (Fig. 3B) is equivalent to the isosceles triangular distribution (Fig. 3C). Alternatively, this isosceles triangular form of the reproduction curve can be obtained by simultaneously varying $p$ from a value of 1.5 to 10.5 days in 1-day intervals as $i$ varies

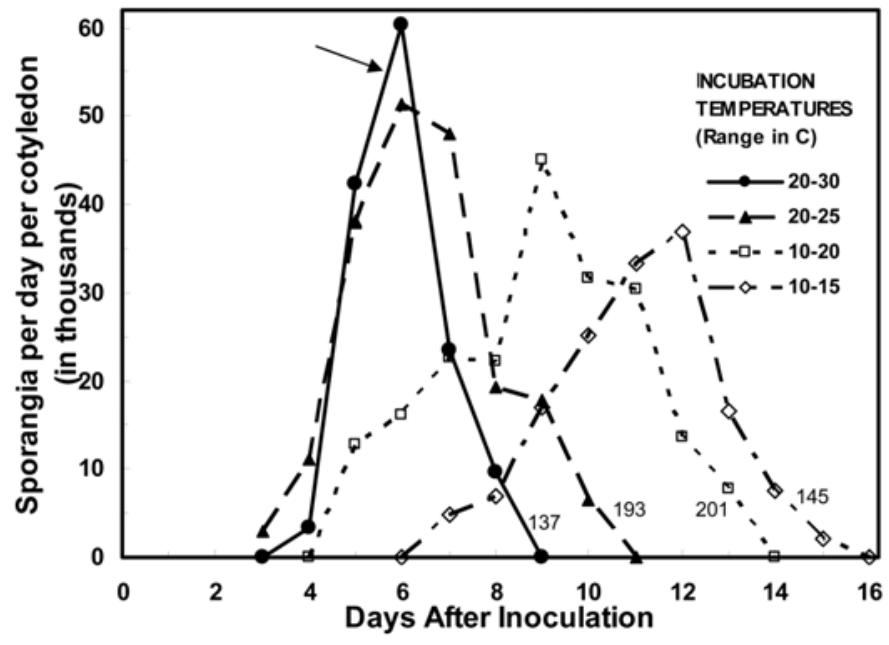

Fig. 1. Daily sporangial productivity of Pseudoperonospora cubensis in cucumber as a function of incubation temperature and time (3). Numbers at the end of each curve represent the total number of sporangia produced (in thousands) for each treatment. 
from 19 to 1 day in 2-day intervals, so that $p$ and $i$ are negatively correlated (Fig. 3F). If the stacked cohorts of Figure 3F are forced to start sporulation at 6 days, one obtains the right-triangular distribution of Figure 3E. Alternatively, we can further shift the stacked cohorts of Figure 3E, such that $p$ varies from a value of 1.5 to 10.5 days in 1-day intervals as $i$ varies from 1 to 19 days in 2-day intervals, so that $p$ and $i$ are positively correlated (Fig. 3D). Note that all the reproduction curves in Figure 3 have the same values for the mean latent period $\bar{p}=6$ days and the mean infectious period $\bar{l}=10$ days.

The same process that created Figure 2 could have been performed just as well for 100 cohorts, in which case the only difference would be that the reproduction curves would have had much smaller steps. In the limit of an infinite number of cohorts, the result is a series of continuous triangular distributions in addition to Vanderplank's SP (Fig. 2, left panel). The area under each curve in Figure 2 is unity. The next question to ask is: How does the shape of these reproduction curves affect the resulting epidemic?

Numerical solution of the Kermack-McKendrick equation. Many mathematical models of plant disease epidemics $(9,27)$ have been shown to be special cases of the more general Kermack-McKendrick epidemic model (KM model) (8). This model is based on the assumption that the reproduction behavior of plant disease lesions is simply a function of lesion age, $\tau$. The
KM model, in turn, is based on the mathematical model of population growth presented by Lotka (4,11-16). The KM model can be expressed as

$$
\begin{aligned}
& \frac{d I(t)}{d t}=\frac{H(t)}{H_{\max }(t)} \cdot \int_{0}^{t} \frac{d I(t-\tau)}{d t} \cdot \Re(\tau) d \tau \\
& H_{\max }(t)=H(t)+I(t) \\
& I(t=0)=I_{0} ; \quad I(t<0)=0
\end{aligned}
$$

where $H(t)$ is the amount of healthy susceptible tissue at time, $t$, $H_{\max }(t)$ is the total amount of susceptible plant tissue, and $I(t)$ is the amount of infected tissue (including latent, $L$; sporulating, $S$; and removed, $R$ ) at time $t$. In order to solve equation 5 numerically, some assumptions are necessary. For the present purposes, we ignored crop growth (5) so that $H_{\max }=H_{0}$, a constant over time. The calculation of infected tissue, $I_{n}$, at time $t=n \Delta t$ is obtained by iterative solution of the following equations:

$$
\begin{aligned}
& \Delta I_{n}=\left(H_{0}-I_{n}\right) \cdot\left[1-\exp \left(-\frac{1}{H_{0}} \sum_{j=0}^{n} \Delta I_{n-j} \cdot D_{j}\right)\right] \\
& I_{0}=I_{0} \\
& I_{-n}=0 ; n>0 \\
& I_{n+1}=I_{n}+\Delta I_{n}
\end{aligned}
$$
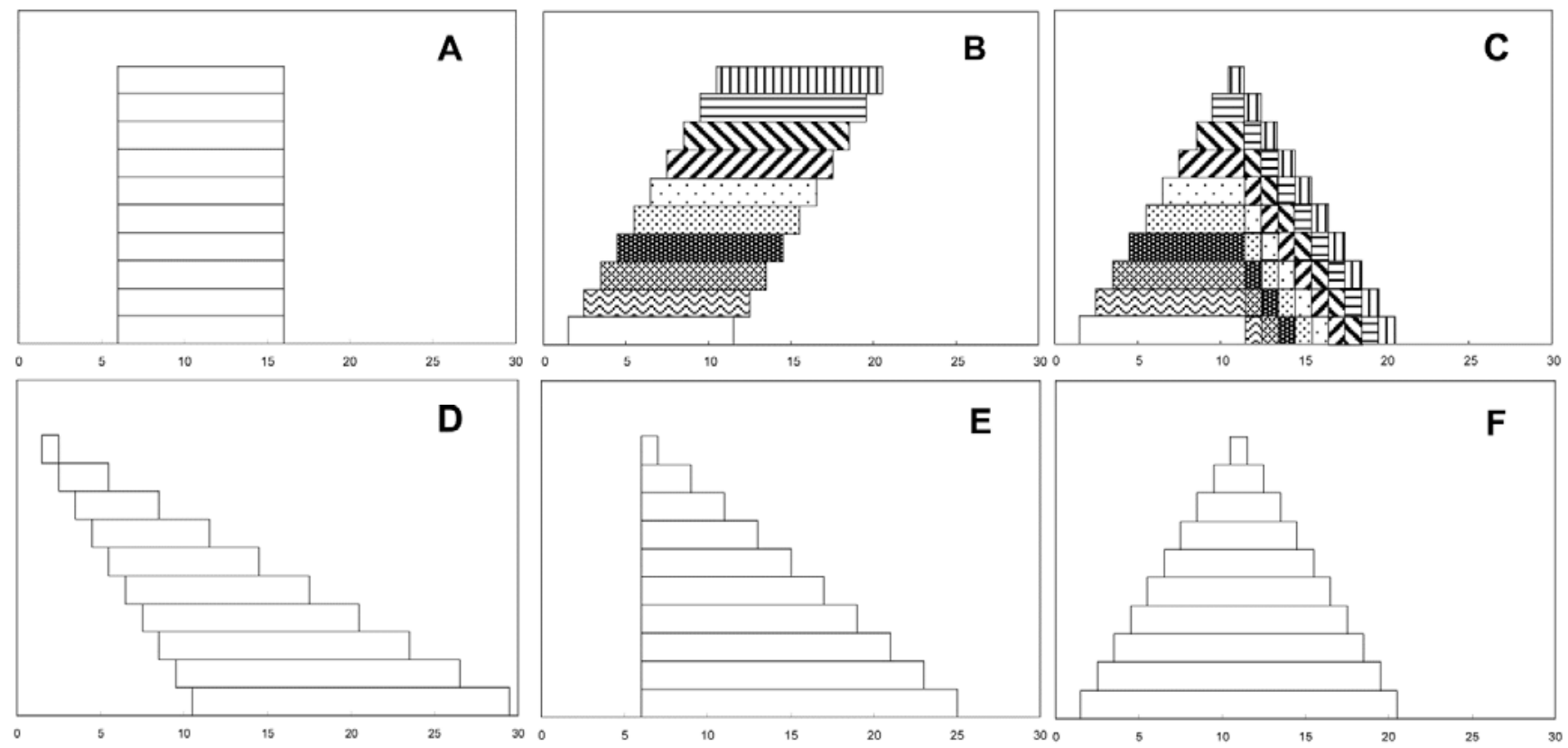

Time from Initial Infection (days)

Fig. 2. A to F, Schematic representations of ensembles of 10 cohorts (equation 3), each with a mean latent period $\bar{p}=6$ days and a mean infectious period $\bar{\imath}=$

\begin{tabular}{|c|c|c|c|c|c|c|}
\hline$\underline{\text { Figure } 2 \text { label }}$ & Shape & $\mu_{\text {ens }}$ (days) & $\sigma_{\text {ens }}^{2}\left(\right.$ days $\left.^{2}\right)$ & $\sigma_{p}^{2}\left(\right.$ days $\left.^{2}\right)$ & $\sigma_{i}^{2}\left(\right.$ days $\left.^{2}\right)$ & $\operatorname{cov}(p, i)\left(\right.$ days $\left.^{2}\right)$ \\
\hline $\mathrm{A}$ & Square pulse & 11 & 8.33 & 0 & 0 & 0 \\
\hline B & Isosceles triangle & 11 & 16.67 & 8.33 & 0 & 0 \\
\hline $\mathrm{C}$ & Isosceles triangle & 11 & 16.67 & 8.33 & 0 & 0 \\
\hline $\mathrm{E}$ & Right triangle & 12.67 & 22.22 & 0 & 33.33 & 0 \\
\hline $\mathrm{F}$ & Isosceles triangle & 11 & 16.67 & 8.33 & 33.33 & -16.67 \\
\hline
\end{tabular}
10 days. A, $p$ and $i$ are constant; $\mathbf{B}, p$ varies from 1.5 to 10.5 days in 1-day intervals and $i$ is constant. $\mathbf{C}$, Same as B but shifted downward to illustrate the isosceles triangular shape; D, $p$ varies from 1.5 to 10.5 days in 1-day intervals as $i$ varies from 1 to 19 days in 2-day intervals, so that $p$ and $i$ are positively correlated; E, $p$ is constant and $i$ varies from 1 to 19 days in 2-day intervals; $\mathbf{F}, p$ varies from 1.5 to 10.5 days in 1 -day intervals as $i$ varies from 19 to 1 day in 2-day intervals, so that $p$ and $i$ are negatively correlated. B, C, and F represent exactly the same reproduction curve.

TABLE 1. Ensemble mean, $\mu_{\text {ens }}$; variance about this mean, $\sigma_{\text {ens }}^{2}$ (equation 4); and the second-order moments of $p$ and $i$ used to calculate these values for the reproduction curves shown in Figure 2 (left) and Figure $3^{\mathrm{a}}$

a Note all of these reproduction curves have a mean latent period $\bar{p}=6$ days and a mean infectious period $\bar{\imath}=10$ days. 
where $D_{j}$ is the potential number of daughter lesions produced by a mother lesion, neglecting the effects of multiple infection (the correction factor) (29), in the time period between $j \Delta t$ and $(j+$ 1) $\Delta t$ after being infected.

The disease progress curves calculated using equation 6 using the reproduction curves shown in Figure 2 (left panel) are plotted in Figure 4 (left panel). The parameters used in these calculations were $I_{0}=1, H_{0}=1,000, R=0.3 \mathrm{day}^{-1}$, and $\Delta t=0.2$ day (see equation 1). There is a 26-day lag between the fastest (Fig. 4, left panel: B, C, and F) and the slowest (Fig. 4, left panel: D) epidemic. This is despite the fact that the reproduction curves have the same values for the mean latent period $\bar{p}=6$ days and the mean infectious period $\bar{l}=10$ days. Obviously, the values of these two reproductive time scales are not good predictors of the time course of the resulting epidemic.

Maintaining the shapes of the triangular reproduction curves shown in Figure 2 (left panel) but adjusting their mean, $\mu_{\text {ens }}$, and variance, $\sigma_{\text {ens }}^{2}$, to be equal to the result for Vanderplank's SP (i.e., $\mu_{\mathrm{SP}}=p+i / 2=11$ days and $\sigma_{\mathrm{SP}}^{2}=i^{2} / 12=8.33$ days $^{2}$ ) results in the normalized reproduction curves shown in Figure 2 (right panel). The disease progress curves calculated using equation 6 using the reproduction curves shown in Figure 2 (right panel) are plotted in Figure 4 (right panel). The parameters used in these calculations were $I_{0}=1, H_{0}=1,000$, and $R=0.3$ day $^{-1}$ (equation 1 ). Obviously, the temporal moments of the reproduction curve are much more important than the latent and infectious periods in determining the time course of the epidemic. It is worth noting that all the epidemics in Figure 4 approach the same asymptotic value. This is due to the fact that every sporulation curve in Figure 2 potentially produces the same exact number of offspring $(20,26)$.

\section{DISCUSSION AND CONCLUSIONS}

I have shown that the use of an average latent period and an average infectious period in the description of fungal reproduction leads to inconsistencies in both interpretation and effect. The root of the problem lies in the meaning of the term "average." When the mean is taken over mother lesions, the average value is equally weighted over the population of initial infections. However, in an epidemic, the contribution of each cohort must be weighted with the total number of its progeny. Thus, assuming

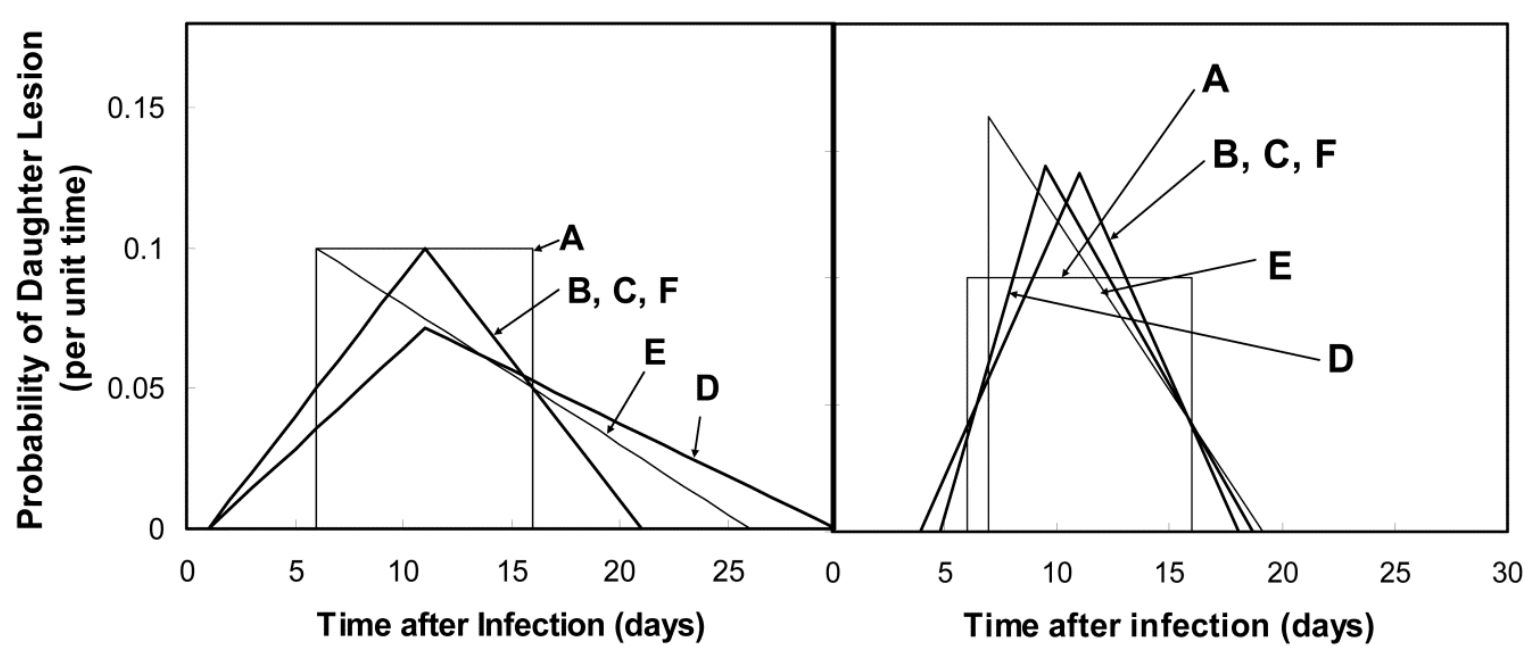

Fig. 3. Left: As the number of cohorts increases indefinitely, the reproduction curves in Figure 2 (Fig. $2 \mathrm{~B}$ to F) approach the above continuous triangular distributions (B to F). Note: the mean latent period, $\bar{p}=6$ days, and the mean infectious period, $\bar{l}=10$ days, for all of these distributions are identical to the result for Vanderplank's square pulse (Fig. 2A). Right: Setting the mean, $\mu_{\text {ens }}$, and the variance about the mean, $\sigma^{2}{ }_{\text {ens }}$, for the various triangular distributions (B to $\mathrm{F}=$ Fig. $2 \mathrm{~B}$ to $\mathrm{F})$ equal to the result for the square pulse $(\mathrm{A}=$ Fig. $2 \mathrm{~A})$, one obtains the above reproduction curves. Details of above calculations are in the online supplemental file.

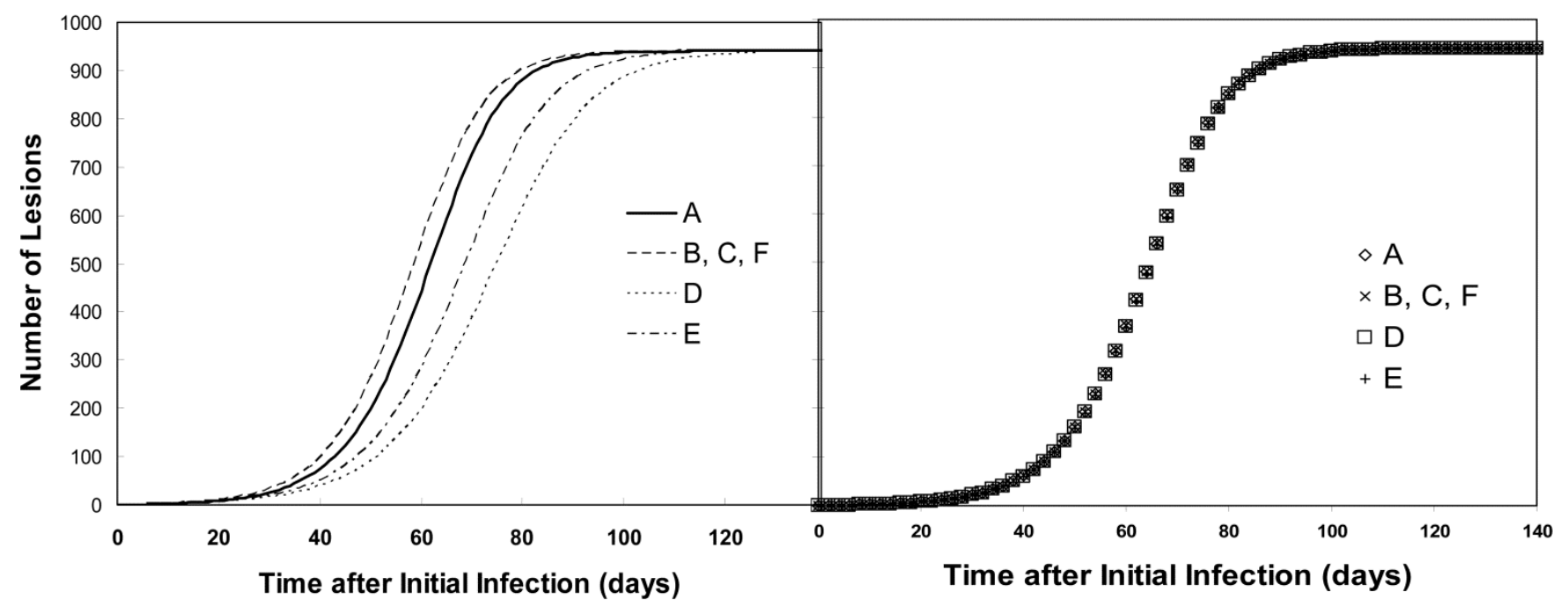

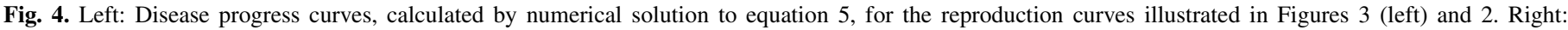

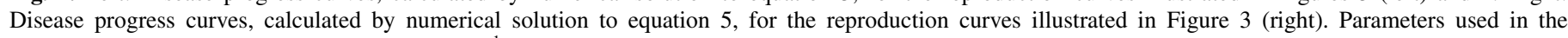
simulation are $H_{0}=1,000, I_{0}=1$, and $R=0.3$ day $^{-1}$ (described in the text). 
that the basic infection rate is constant, lesions that sporulate over a longer period of time become more important in describing the resulting epidemic. This leads to an ensemble mean time of reproduction and variance about this mean, which are complex in nature and not very intuitive when expressed in terms of latent period and infectious period of the mother lesions. In addition, estimates of these quantities can rarely be obtained by direct experiment because the reproductive history of individual infections must be followed separately. In contrast, the well-defined and easily extracted temporal moments of spore production curves provide an objective description of the course of progeny production, which is directly related to the dynamics of the epidemic $(4,11,21,24)$.

\section{ACKNOWLEDGMENTS}

I thank D. E. Aylor for helpful discussions and constructive criticism during the preparation of this manuscript.

\section{LITERATURE CITED}

1. Bashi, E., and Aust, H. J. 1980. Quality of spores produced in cucumber powdery mildew compensate for their quantity. Z. Pflanzenkrankh. Pflanzenschutz 87:594-599.

2. Cohen, Y., and Rotem, J. 1971. Rate of lesion development in relation to sporulating potential of Pseudoperonospora cubensis in cucumber. Phytopathology 61:265-268.

3. Cunniffe, N. J., Stutt, R. O. J. H., van den Bosch, F., and Gilligan, C. A. 2012. Time-dependent infectivity and flexible latent and infectious periods in compartmental models of plant disease. Phytopathology 102:365-380

4. Dublin, L. I., and Lotka, A. J. 1925. On the true rate of natural increase. J. Am. Stat. Assoc. 20:151:305-339.

5. Ferrandino, F. J. 2008. The effect of crop growth and canopy filtration on the dynamics of plant disease epidemics spread by aerially dispersed spores. Phytopathology 98:492-503.

6. Heagle A. S., and Moore, M. B. 1970. Some effects of moderate adult plant resistance to crown rust of oats. Phytopathology 60:461-466.

7. Kato, H., and Kozaka, T. 1974. Effect of temperature on lesion enlargement and sporulation of Pyricularia oryzae on rice leaves. Phytopathology 64:828-830.

8. Kermack, W. O., and McKendrick, A. G. 1927. A contribution to the mathematical theory of epidemics. Proc. R. Soc. A 115:700-721.

9. Leonard, K. J., and Mundt, C. C. 1984. Methods for estimating epidemiological effects of quantitative resistance to plant diseases. Theor. Appl. Genet. 67:219-230.

10. Lewontin, R. C. 1965. Selection for colonizing ability. Pages 77-91 in:
Genetics of Colonizing Species. H. G. Baker and G. L. Stebbins, eds. Academic Press, London, New York.

11. Lotka, A. J. 1907. Studies on the mode of growth of material aggregates. Am. J. Sci. Ser. 4 Vol. 24:199-216.

12. Lotka, A. J. 1922. The stability of the normal age distribution. Proc. Natl. Acad. Sci. USA 8:11:339-345.

13. Lotka, A. J. 1928. The progeny of a population element. Am. J. Epidemiol. 8:6:875-901.

14. Lotka, A. J. 1929. Biometric functions in a population growing in accordance with a prescribed law. Proc. Nat. Acad. Sci. USA 15:10:793798.

15. Lotka, A. J. 1939. On an integral equation in population analysis. Ann. Math. Stat. 10:2:144-161.

16. Lotka, A. J. 1942. The progeny of an entire population. Ann. Math. Stat. 13:117-118.

17. McGregor, A. J., and Manners, J. G. 1985. The effect of temperature and light intensity on growth and sporulation of Puccinia striiformis on wheat. Plant Pathol. 34:263-271.

18. Meats, A. 1971. The relative importance to population increase of fluctuations in mortality, fecundity and the time variables of the reproductive schedule. Oecologia 6:223-237.29.

19. Mehta Y. R., and Zadoks, J. C. 1977. Uredospore production and sporulation period of Puccinia recondita f. sp. triticina on primary leaves of wheat. Neth. J. Plant Pathol. 76:267-276.

20. Metz, J. A. J. 1978. The epidemic in a closed population with all susceptibles equally vulnerable: some results for large susceptible populations and small initial infections. Acta Biotheor. 27:75-123.

21. Metz, J. A. J., and Diekmann, O. 1986. The Dynamics of Physiologically Structured Populations. Lecture Notes in Biomathematics 68. SpringerVerlag, Berlin

22. Painter, P. R., and Marr, A. G. 1967. Inequality of mean interdivision time and doubling time. J. Gen. Microbiol. 48:155-159.

23. Papastamati, K., and van den Bosch, F. 2007. The sensitivity of the epidemic growth rate to weather variables, with an application to yellow rust on wheat. Phytopathology 97:202-210.

24. Pielou, E. C. 1969. Pages 45-48 in: An Introduction to Mathematical Ecology. John Wiley and Sons, New York.

25. Savary, S., De Jong, P. D., Rabbinge, R, and Zadoks, J. C. 1990. Dynamic simulation of groundnut rust: a preliminary model. Agric. Syst. 32:113141.

26. Segarra, J., Jeger, M. J., and van den Bosch, F. 2001. Epidemic dynamics and patterns of plant diseases. Phytopathology 91:1001-1010.

27. Teng, P. S., and Close, R. C. 1978. Effect of temperature and uredinium density on urediniospore production, latent period, and infectious period of Puccinia hordei Otth. N.Z. J. Agric. Res. 21:282-296.

28. Vanderplank, J. E. 1963. Plant Disease: Epidemics and Control. Academic Press, Inc., New York.

29. Vanderplank, J. E. 1965. Dynamics of epidemics of plant disease. Science $147: 120-124$.

30. Vanderplank, J. E. 1975. Principles of Plant Protection. Academic Press Inc., New York. 